\title{
Lessons learnt from CSCL to enrich E-Learning
}

\author{
M. Beatrice Ligorio \\ University of Bari, Dept. of Psychology, \\ Palazzo Ateneo, Via Crisanzio 1 - 70100 Bari - Italy \\ bealigorio@hotmail.com \\ Roberto Cordeschi \\ University of Salerno, Dept. of Communication Sciences \\ Via Ponte Don Melillo 84084 - Fisciano (SA) - Italy \\ cordeschi@caspur.it
}

\begin{abstract}
Computer Supported Collaborative learning (CSCL) is nowadays well mature and it is able to set guide-lines to enrich e-learning. On its side, e-learning does not fully exploit its potentialities yet. Suggestions from CSCL could be useful to empower the presence on the elearning on the market and would foster a vision of learning as not a simple further "commercial product".

The European Commission funded many projects involving several countries, numerous students, teachers and researchers, and a variety of software. During the presentation a few European projects, considered as best practices, will be shortly discussed. Through the results gathered, guide-lines are drawn to improve e-learning, concerning: a) understanding of the users; b) blended learning; c) learners' empowerment.
\end{abstract}

Keywords: CSCL, educational theories, e-learning at school

\section{INTRODUCTION}

Computer Supported Collaborative Learning (CSCL) is a more innovative branch of the traditional ICT stream of research. This new branch underlines the use of computers as tools and artifacts sustaining a particular form of learning, namely collaborative learning within a community of learners. The advantages of collaborative learning over individual learning are broadly acquired. The term "collaborative learning" refers to a general method of working, where learners at various performance levels, work together in groups of different size, toward a common goal. Every individual is responsible for the other's learning as well as their own. Thus, the success of one student helps other students to be successful.

According to Johnson and Johnson (1986) [1], there is persuasive evidence that cooperative teams achieve at higher levels of thought and retain information longer than students who work as individuals. Collaborative learning gives students an opportunity to engage in meaningful discussion, take responsibility for their own learning, and thus become critical thinkers [2].

Slavin (1990) [3] points out that collaborative learning is able to intertwine cognitive and social aspects of learning. In this way learning is no longer an individual fact but strongly involves the group and the context where learning occurs. By moving the focus to the social context, also the artefacts used to promote collaborative learning became important. In particular, collaborative learning can be promoted by tools enhancing communication and interaction, the two factors underling the social dimension of learning.

CSCL studies all kinds of situations where technology covers the role of an artefact promoting collaborative learning. As Lipponen (2002) [4] has clearly remarked, within the CSCL framework there are many different controversial aspects. For example, it includes studies with great differences in terms of the size of the sample, the duration of the research, the topic and task students work on, the unit of the analysis (ranging from students dyads to the entire classroom or even larger community). Even with those unsolved diversities, or maybe thanks to them, CSCL contributes to the enhancement of the understanding of collaborative learning at least in two ways: 
- By providing new occasions for more sophisticated learning: new and higher process of thinking, reasoning, and interaction can be activated when technology is an active part of the learning scenario;

- By fostering the design of technology deliberately aimed at supporting collaborative learning, new interfaces and functionalities are designed that would not have existed if not serving that function.

Using these particular perspectives, CSCL has today gathered enough experiences, data, and reflections to give valuable contributions to better understand and implement successful e-learning experience. Suggestions are indeed needed since e-learning seems to not besiege the market as it could. One of the reasons of e-learning failure could be the wrong vision of learning and the erroneous interpretation of its potential users. CSCL experiences have great amounts of data regarding precisely the learners' needs, learning processes supported by educational technology and models of learning. These data can be at the service of e-learning developers and disseminators.

\section{REVIEW OF SOME CSCL BEST PRACTICE}

In order to adduce evidence to the statement that CSCL experience is able to improve e-learning, three projects funded by the European Commission will be briefly reported. The three projects were selected based on the following criteria:

- Fairly large size in terms of students, teachers, researchers, and schools sites involved;

- Cross-national nature, which is considered as one of the strongest added value of using e-learning;

- Broad range of educational activities covering several curricula;

- Results gathered can be discussed in terms of changes to be implemented into the platform.

The three projects described in the following can be considered as best practices in the field of CSCL [5]. Furthermore, the lesson learnt from these researches seems to offer interesting suggestions to how elearning can profit from the CSCL experience.

\subsection{Cl-Net}

Cl_Net (Computer Supported Collaborative Learning Networks) was a two-year project funded in 1998 by the European Commission under the TSER program. The main objective of this project was to compare different computer-supported collaborative learning networks (CLNs) looking for advantages and obstacles when implemented in real education contexts. The central question of the project was: How can effective knowledge building in CLN's be supported in European primary and secondary education? Five European countries were involved (Belgium, Finland, Greece, Italy, The Netherlands) with almost 600 students from primary (age 10-12), secondary (age 13-16), and vocational education (age 18-24), together with 25 teachers from 20 schools. Different types of technological settings were used and compared [6].

The technological platform used by the participating sites differed as to (a) content, (b) curriculum, and (c) target group characteristics. For example, WorkMates was a common tool for information delivery and communication, used by the research group for exchange of documents and project-bound information. Our World - a data base for classifying environmental data -, Discovery Your Town - a multimedia game to learn about the Middle Age- , and Our Castle - a web-site to built an hypertext - (all used by the Italian sites) are instances of highly content oriented and multimedia software, referring to both different groups of children and specific curriculum content. Computer Supported Intentional Environment (CSILE) and Web Knowledge Forum (WKF) [7] are discussion forums consistent with the notion of "co-construction" of knowledge, using very open software to support cooperation and collaboration in many subject-matter domains, like arithmetic and literacy. The quality of the software highly depends upon the quality of both the information put into the database and the concomitant support offered by teachers and peers.

This project produced two types of results: case studies and comparative results. Eight different case studies were carried out within the Cl_Net project. Each case differed in terms of school structure and culture, teachers' attitude and training, general role of the school in the society, and so on. These diversities allow reflecting upon the non-homogeneous state of European education under the institutional point view. In fact, 
if the theoretical background of the project (primacy of collaborative learning over individual learning, socioconstructivism approach, CSCL framework) could be shared by all the countries involved, the implementation was very sensitive to local factors such as curriculum structure, timetable, general organization of the school, teachers attitude toward technology. All these factors strongly impact reasons for successes or failure of the CSCL implementation in the classroom. For example, better results were often gathered when teachers were more inclined to introduce innovation into their classroom and when the whole school (the principal and the colleagues) was willing to foster the project.

The comparative results were composed by three types of instruments used by all the sites: a) Communications protocols between students and between students and teachers; b) Questionnaires and interviews to teachers and students; b) Tests measuring cognitive, metacognitive, and motivational effects of the project. The latter type of instrument was administrated in all the sites as pre and post test, thus it could be used to generalize the results obtained. In particular, consistent evidence was found suggesting that during the course of the project many students showed less emphasis in superficial engagement and more interest in real collaboration. Motivation increased in almost all test sites.

The findings gained from the different test-sites where cognitive effects were measured and reported, show significant advantages of introducing CSCL in classrooms. An improvement of the score was obtained by the students participating and answering to standardized test scores for curricular domains, such as language and mathematics as well as process-oriented measures, like quality of question-raising, and depth of explanation. Also interesting findings were detected from the analysis of the metacognitive questionnaire, especially in those sites where the project lasted the whole academic year.

\subsection{Euroland}

Euroland was a one-year project funded in the 1999 by a "Training and Mobility of Researchers" (TMR) Marie Curie Research Training Grant. The project sought a strong collaboration between Italy and The Netherlands. Seven schools were involved ranging from primary to high school. A total of 268 students, 13 teachers and a cross-national research group composed by 8 researchers participated very actively. Two of those researchers acted as tutors on-line always connected during the weekly synchronous meeting scheduled. The aim of this project was to sustain and develop a virtual community able to integrate activities on-line (both synchronous and asynchronous) with activities in the classroom.

The virtual community populated a learning environment composed by a three-dimensional virtual world, created by a software named Active Worlds (AW) (www.activeworlds.com) combined to the Web Knowledge Forum, designed by Scardamalia and Beretier (1994) [7].

AW is desktop, internet-based, user-oriented software and it belongs to the non-immersive type of virtual reality. Within the virtual worlds built with AW, users can walk through, navigate, and fly over threedimensional (3D) objects. The presence of a user is made visible thanks to "Avatars," which are animated 3D objects that may range from a puppet to a penguin or some other animal. AW supports mainly synchronous communication via text-based chat and visual Avatars and $3 \mathrm{D}$ objects interactions and manipulation. The integration of AW with $\mathrm{KF}$ is easy under the technical point of view: it is enough to build a 3D object with an active link to KF. The figure here below shows how Aw and KF look when running together. 


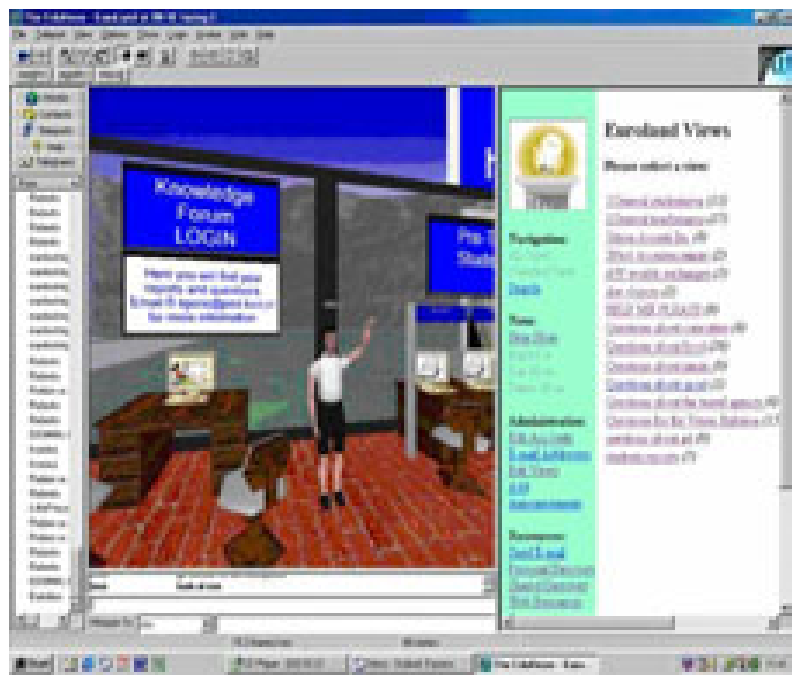

FIGURE 1: The computer screen as it appears when AW and KF are running at the same time

A complex and rich internet based environment was obtained, and different types of communication were possible: text-based (more in the forum) versus visual (3D objects); and synchronous (chat) versus asynchronous (forum). This mix allowed fulfilling different types of needs, fostering the participation of people with different capabilities and competencies [8].

The final result of this project was a virtual world full of houses with educational content. In particular it was possible to distinguish:

- Cultural houses: the house of music, art, food, sport, and a travel agency. In the construction of those houses it was attempted to include subject-matter issues. Since those houses were considered as the most relevant under the curricula point of view, the researchers wanted to apply to them the collaborative learning strategies, not only while working in the classroom but also when working at a distance. The group in charge of a specific cultural house (let's say the house of food) had to build the house of the other country. That is to say that the Italians had to build the house of Dutch music, art, food, and sport, while the Dutch students were taking charge of the Italian ones. In this way each group was positively and genuinely interdependent [9] upon the partner at the distance. In fact, the virtual partners were not simply those to whom the final products were shown, but competent and expert sources of information that could be involved in all the phases of the project, from the planning to the decoration of the houses.

- Individual houses where each group could represent its own identity.

- Social spaces where the community could meet to talk and discuss (i.e., the Conference room) or to share relevant information about the project (i.e. the Welcome area built by the researchers).

This organization of the space allows reflecting upon how a virtual environment should be structured in order to sustain collaboration both in the classroom and on-line, and how to foster the collective and social dimension along with the individual dimension, both necessary to develop efficient collaboration strategies [3].

The construction of digital identities was quite crucial in this project. Participants spent a lot of time on choosing nicknames and avatars and on talking about them. Often the help of other connected users was requested during the process of constructing digital identities. This data lead us to consider digital identities as great "control levers" of learning into virtual environments [10]. The educational nature of this virtual space implies that positioning - to use a term introduced by Harrè and Van Langenhove (1991) [9] -shown and "built" contribute to the construction of participants' image of themselves as learners. Telling who we are and who we are able to be is a "dialogical" game [11] that can make the learning experience more interesting and richer.

The analysis of this project highlighted the following crucial issues:

a) The virtual environment was easy under the technical point of view, nevertheless lots of the time was allocated to train teachers about the educational way of using the environment. 
b) The playful dimension given by the availability of $3 \mathrm{D}$ objects and the chat, made the environment look like an entertainment place. This raised students' motivation but represented a worry for teachers, for whom learning environments had to be serious.

c) Although cross-national communication seems to be an added value of electronic environment, it is not easy to take place in an efficient way. A lot of support was needed by experts and tutors on-line. Students and teachers were often frustrated by the initial not spontaneous interaction, despite the motivation and the excitement of meeting people from another country. The initial inhibition of expressing themselves in other language needs to be overcome.

d) Specific didactical material needs to be developed. Multi-disciplinary and innovative content seems to be more suitable to electronic environments but, how to develop and integrate this material in the existing curricula?

\subsection{Itcole}

ITCOLE (www.euro-cscl.org/site/itcole) was a two-year project funded by the European Commission within the $5^{\text {th }}$ framework. Four European countries participated by providing experimental sites, 84 teachers (experienced and novice) and 1413 students were involved from primary, secondary and university level. 66 local projects were carried out within the main program, some of them were designed as cross-classroom collaboration and others required cross-national collaboration. The ITCOLE main objective was to design a pedagogically meaningful modular knowledge-building environment, Synergeia, which supports students' joint efforts, tailored to different European school cultures [12].

Synergeia (http://bscl.gmd.de/bscl2) was developed through two testing phases: phase 1 aimed at providing information of the participating teachers' first impressions of Synergeia, and their conceptions on pedagogical principles. The data gathered during the phase 2 provided information about the projects carried out in classrooms, teachers' and students' experiences during, and after the first projects conducted with Synergeia.

Beside quantitative data concerning the number of notes and materials posted on Synergeia, this project produced many types of teachers' and students' questionnaires, interviews, and users stories in order to have a clear picture of what students and teachers experienced.

The teachers in all four countries were convinced that the use of electronic learning environment supports collaboration among students. However, they concluded that for students to be able to build new knowledge was a complex process, especially when they were not explicitly guided by teachers. Furthermore, they consider well thought-out face-to-face activities as absolutely needed to support such complex process.

Despite the fact that teachers thought that collaboration was not 'natural' for students, students themselves reported that they collaborated successfully within the project. This conclusion was stronger for secondary education than for primary education.

About the software, it was a general opinion that it supported the process of collaborative learning in an effective way. This was caused by the fact that the process was visible to everyone, and products and ideas of others could easily be seen because they were posted on the web platform. However, many teachers claimed that deeper levels of collaboration and shared development of products need extra support and guidance. Furthermore, more specific training on collaborative learning was required.

Students reported that they enjoyed collaborating. Many of them improved their feeling of self-respect due to their contributions to collective discussions. Together with the other findings, it seems that working in the electronic learning environments stimulates community building, although this is not an automatic process. Both teachers and students recognize that new skills are needed in order to foster the process of community building.

Many aspects seem to influence the teachers' stance relative to this project, such as their previous experience with CSCL and the school level. Primary school teachers were in general more successful than secondary school teachers. In general, successful CSCL learning projects depend on enough training for teachers, both on pedagogical and technical aspects; enough time for teachers to prepare the classroom projects; and including the project within the curriculum and at the same time keeping a cross-curricula dimension. Teachers with experience on CSCL could dedicate more time and effort to scaffold the processes within the CSCL-projects, where novice teachers needed time to get acquainted with both a new pedagogical model and a new process. Generally, primary school teachers have more time to implement projects, which may explain that they are relatively more successful. Another factor is the fact that students in secondary education do not work usually with the collaborative learning paradigm. They might be more reluctant to embrace this new approach, where primary students carry less 'luggage' of traditional education and are more open to new kinds of education. 


\section{CONTRIBUTIONS TO E-LEARNING}

One of the risks of a large scale e-learning diffusion is to treat education as a phenomena submitted to the same economical laws that apply to any commercial product. Introducing Internet and intranet technologies into educational contexts offer tremendous opportunities to bring learning into the mainstream of business.

Based on the results discussed above and gathered by three European best practices of CSCL we can outline the conditions under which e-learning can be more than a new business:

- Platforms must be able to support pedagogical theories, in particular collaboration at a distance, knowledge building, reflective thinking, and virtual community building. Internet based platforms will be really used as tool for learning only when before building the technical tools there is a clear idea of what type of learning we want to promote. This may mean a different location of the economical investment. When elearning platforms are designed on users' needs, more time and energy is needed at the very initial stage of the planning, instead of the stage of dissemination and distribution of the platform. In fact, at the outset of planning a platform it is necessary to build a few bridges: between users and educational researchers, between educational researchers and computer scientists. New professional figures with mixed competences could be helpful: educators with technical competences, computer scientists specialized on educational software, users trained on knowing what can be required to an e-learning platform; school advisors with knowledge of economical aspects concerning software.

- Platforms have to be rich and flexible. Although the presence of several tools can cause an initial confusion, we experienced that users enjoy activities where they can use different tools and where the environment can be tailored on individual or groups' needs. Most of the time initially users do not have a clear idea of how to use a new tool. The tool itself, if well designed, should suggest new activities that could not be done without it. E-learning platforms could provide the most innovative news of the Internet but, must importantly, should suggest educational uses.

- Pedagogical content should be innovated along with the technology use. The constant and successful use of e-learning is necessarily going to modify the pedagogical models that initially inspired it. If collaboration at a distance, knowledge building, reflective thinking, and virtual community building proved to be the most valuable educational frameworks within which to design and implement e-learning, those models show their limits and weak sides when looking deeper into the results gathered by best practices we discussed. Collaboration is not easy to achieve, especially when partners with different competencies, language, cultural backgrounds are involved. Knowledge building appears to be possible only when technical problems are solved and when teachers are experienced enough. Reflective thinking and metacognitive skills are impacted only when projects have a fairly long duration (at least one academic year). Community building and processes through which digital identities and positioning are formed should be better taken into account.

It is now possible to outline some guidelines to improve e-learning based on CSCL lessons:

- Better understanding of the local settings and of participants' implicit expectations. E-learning implementation can be difficult due to the diversity of participants. The effort on planning and implementing e-learning does not have to be focused on reducing diversity but on using diversity as a resource. Specific spaces should be allocated, within the e-learning platform, for participants to describe and discuss their own contexts and to find ways to value their local resources.

- Blended learning. E-learning is not just about using the latest technology in order to replace the classroom. Nor is it about posting content on the Web to be downloaded or read by someone else. Elearning provides a new set of tools that can add value to all of the traditional learning modes, from classroom experiences to learning on the job. The more e-learning is blended with on-the-site activities, the more users will feel involved and will contribute in enriching the platform.

- Learners considered as able to create new knowledge. This may require a theoretical revision of the idea of "knowledge building" society [13]. Replacing "creation" with "building" is not a mere play of words. It is a serious attempt to replace rational/scientific thinking with more space for intuition, creativity, and ability to think ahead [14]. Those skills are soon going to have a commercial and economical value also for training and work-places. Standard psychological theories view creativity as arising largely from the unique or 
extraordinary characteristics of individuals (e.g., mental processes, background knowledge, intellectual style, personality, and motivation) giving voice to social attitudes and beliefs about the folklore of such terms as the lone genius, brilliant inventor, estranged artist, or ruthless entrepreneur. In fact, any creative product emerges from a unique coincidence of individual intellectual abilities; the nature and relative sophistication of a scientific, artistic or entrepreneurial domain; the complexity and structure of the field of legitimization; and the distribution of power and resources within a group, community or society. E-learning has to provide this type of opportunity by empowering the users, forming new and complex groups, putting in contact diverse cultures and attitudes. The accent on creativity is a natural consequence of the de-materialization of the modern economy, of the transition from the industrial to the post-industrial society, where the first is based on the information and the latter on the ability to create. This implies that human resource is more important than economical resource and that a learner-centred perspective is a key factor to lay out successful virtual learning environments.

\section{REFERENCES}

[1] Johnson, R. T. and Johnson, D. W. (1986) "Action research: Cooperative learning in the science classroom", Science and Children, 24, pp. 31-32.

[2] Totten, S., Sills, T. , Digby, A. and Russ, P. (1991) Cooperative learning: A guide to research. New York: Garland.

[3] Slavin, R. (1990) Cooperative learning: Theory, research and practice. Englewood Cliffs, NJ: Prentice Hall,

[4] Lipponen, L. (2002) Exploring the foundations for computer-supported collaborative learning: In G. Stahl (Ed.) Proceedings of CSCL 2002: The Fourth International Conference on Computer-Supported for Collaborative Learning, January 7-11, 2002, Boulder, Colorado (USA), 72-81.

[5] Talamo, A., Ligorio, M.B. and Simons, R.J. (2003) "How do we innovate by researching?" In Mario Barajas (Ed.) Learning innovations mediated by ICT: A socio-economic perspective. University of Barcellona (ES), pp. 85-98

[6] Van der Meijden, H., Simons, R.J. and De Jong, F. (2000) (Eds). Computer-supported collaborative learning networks in primary and secondary education. Final Report for the European project CL_Net no 2017, University of Nijmegen (NL).

[7] Scardamalia, M. and Bereiter, C. (1994) "Computer support for knowledge-building communities", The Journal of the Learning Sciences, 3, pp. 265-283.

[8] Ligorio, M.B. (2001) "Integrating communication formats: synchronous versus asynchronous and text-based versus visual", Computers \& Education. Vol. 37/2, pp. 103-125

[9] Salomon, G. (1998) "Novel constructivist learning environments and novel technologies: some issues to be concerned with" Research Dialogues in Learning and Instruction, 1 (1), pp. 3-12.

[9] Harrè, R. and Van Langenhove, L. (1991) "Varieties of positioning", Journal for the Theory of Social Behaviour, 21, pp. 393-407.

[10] Talamo, A. and Ligorio, M.B. (2001) "Strategic identity in the cyberspace", Journal of CyberPsychology and Behavior, G. Riva, C. Galimberti (Eds.) Special Issues "The mind and the Web: Psychology in the Internet age", Vol. 4, n. 1, pp.109-122

[11] Hermans, H.J.M. (2001) "The dialogical self: toward a theory of personal and cultural positioning" Culture \& Psychology, 7(3), pp. 243-281.

[12] Ligorio, M.B. and Veermans, M. (in press, 2005) "Perspectives and patterns in developing and implementing international web-based Collaborative Learning Environments", Computers \& Education

[13] Von Krogh, G., Ichijo, K. and Nonaka, I. (2000) Enabling knowledge creation. Oxford: Oxford University Press.

[14] Runco, M. A. and Albert, R. S. (1990) (Eds.) Theories of creativity. Newbury Park, CA: Sage. 